\title{
PRESENTACIÓN DEL DOSSIER: Aspectos Económicos de la Propiedad Intelectual
}

Dossier's Presentation: Economics of Intellectual Property

\author{
Sophia Espinosa Coloma (COORd.) \\ Universidad San Francisco de Quito
}

\begin{abstract}
El Acuerdo sobre los Aspectos de los Derechos de Propiedad Intelectual relacionados con el Comercio (ADPIC) fija los estándares mínimos para la protección de los derechos de propiedad intelectual, con el fin de reducir las distorsiones e impedimentos en el mercado internacional entre los países miembros de la Organización Mundial de Comercio. De esta manera, el ADPIC homologa los parámetros básicos de protección de la normativa internacional de propiedad intelectual para facilitar el comercio internacional, tal como señala en su preámbulo:
\end{abstract}

Deseosos de reducir las distorsiones del comercio internacional y los obstáculos al mismo, y teniendo en cuenta la necesidad de fomentar una protección eficaz y adecuada de los derechos de propiedad intelectual y de asegurarse de que las medidas y procedimientos destinados a hacer respetar dichos derechos no se conviertan a su vez en obstáculos al comercio legítimo (1994, p. 342).

No obstante, cuando se suscribió el ADPIC en 1994, no sólo se buscaba un tratado que armonizara el comercio internacional, sino que además promoviera el desarrollo tecnológico de todos los países miembros. De ahí que el Artículo 7 establezca como objetivo la necesidad de que estos derechos de propiedad intelectual contribuyan a la innovación, difusión y transferencia tecnológica, buscando el bien de los titulares de derechos de propiedad intelectual y de sus usuarios1. El origen de esta disposición se encuentra en una propuesta presentada al Grupo de Negociación de la Ronda Uruguay por las delegaciones de Argentina, Brasil, Chile, China, Colombia, Cuba y Egipto, India, Nigeria, Perú, Tanzania y Uruguay. El Artículo 7 está fuertemente influenciado por los objetivos contenidos en la propuesta que buscan un equilibrio entre las necesidades de desarrollo económico, social y tecnológico y los derechos otorgados a los titulares de derechos de propiedad intelectual ${ }^{2}$.

Los países en desarrollo trataron de garantizar que el otorgamiento de derechos de propiedad intelectual no sólo beneficiara a los titulares de estos derechos, sino también que fomentara otras actividades para promover el bienestar social y la transferencia de tecnología. Este artículo pretende equilibrar los derechos e intereses públicos y privados para la conveniencia de la sociedad. "This general provision was proposed by developing countries and intent to safeguard the proper balance between private rights and public goods against one-sided and detrimental effects that IPRs may have, and in order to promote their use as incentives for foreign investment and technology transfer" (Biber-Klemm, Cottier et al., 2006, p. 75).

\footnotetext{
${ }^{1}$ Art. 7 "La protección y la observancia de los derechos de propiedad intelectual deberán contribuir a la promoción de la innovación tecnológica y a la transferencia y difusión de la tecnología, en beneficio recíproco de los productores y de los usuarios de conocimientos tecnológicos y de modo que favorezcan el bienestar social y económico y el equilibrio de derechos y obligaciones".

${ }^{2}$ Más información sobre las negociaciones del GATT (Group of Negotations on Goods) que dieron origen al ADPIC (o TRIPS por sus siglas en inglés), puede consultarse en la Comunication from Argentina, Brazil, Chile, China, Colombia, Cuba, Egypt, India, Nigeria, Peru, Tanzania and Uruguay (1990).
} 
Actualmente, a más de veinte años de la entrada en vigencia del ADPIC, es fundamental determinar hasta qué punto este sistema de derechos ha cumplido efectivamente con los objetivos contenidos en el Artículo 7. De ahí que en los últimos años se haya intensificado el debate sobre el impacto económico que ha tenido la implementación del sistema de propiedad intelectual (Wipo, 2009). La necesidad de estudiar con mayor detenimiento el efecto de los derechos de la propiedad intelectual en la economía de las naciones alcanza una mayor intensidad, por el hecho de que hoy vivimos en una economía del conocimiento. Es decir, una economía que está basada en activos intangibles, derechos de propiedad intelectual y, por ende, creaciones del intelecto humano. La transformación de los mercados globalizados, por medio de la innovación tecnológica y de los negocios, ha hecho que el valor de mercado de las empresas pase a fundamentarse en activos intangibles en lugar de tangibles: las estadísticas muestran que en 1975 el valor de mercado de las empresas era de un 83\% activo tangible y un 17\% activo intangible; hoy en día, el valor de los intangibles ocupa el $84 \%$ del valor total de mercado (Stathis, 2015).

Tradicionalmente, economistas y expertos legales han sugerido que la adopción de sistemas fuertes de derechos de propiedad intelectual tiene un impacto directo en el crecimiento económico. Estos señalana, asimismo, qué estándares más fuertes de propiedad intelectual traen como resultado una mayor investigación y desarrollo, y un aumento de la inversión y transferencia tecnológica (Gutterman, 1993, pp. 119-120). Sin embargo, es fundamental que nos preguntemos hasta qué punto estos efectos se dan en todos los países que han incorporado de una u otra manera el ADPIC, es decir, debemos interrogar si, la mera adopción de estándares mínimos de protección en esta área, da como consecuencia absoluta los mencionados resultados.

En ese sentido, es cierto que para muchos países, especialmente los llamados países desarrollados, los sistemas fuertes de propiedad intelectual tienen un efecto positivo en el crecimiento económico. Esto se debe a que los regímenes más fuertes de propiedad intelectual aumentan el valor de los activos que son objetos de protección. Por lo tanto, si consideramos que la mayoría de los titulares de derechos de propiedad intelectual proceden de países industrializados, estos países y sus industrias se beneficiarían del sistema. Por ello, la aplicación del ADPIC tuvo un efecto positivo para los países desarrollados como los Estados Unidos de América: "the biggest exporter of proprietary rights and thus the largest recipient of royalties and license fees has traditionally been the United States. Total recipients of royalties and license fees increased from an average of $\$ 6.7$ billion in 1980-82 to an average of $\$ 23.3$ billion in 19931995” (Primo Braga, Fink y Sepulveda, 2000, p.18).

Sin embargo, los efectos de un sistema de propiedad intelectual más fuerte en los países en vías de desarrollo y subdesarrollados no son tan positivos a corto plazo. El primer efecto de esto es una transferencia de riqueza de los países pobres a los países industrializados que poseen los derechos de propiedad intelectual (Abbot, 1996). Este es un resultado lógico del sistema, ya que los consumidores de países en vías de desarrollo tienen que pagar derechos de licencia o el precio real de la obra patentada o derechos de autor a los titulares de derechos de propiedad intelectual.

De ahí que los beneficios como el aumento de la investigación y el desarrollo y la transferencia de tecnología sean relativos. En primer lugar, para hablar de investigación y desarrollo, debemos considerar si los países en desarrollo tienen la capacidad de participar en esas actividades. Es decir, tenemos que evaluar si el recurso humano del país en cuestión tiene la formación académica y la experiencia para trabajar en nuevas invenciones. Asimismo, tenemos que determinar si tienen acceso suficiente a nueva tecnología para permitir que investigadores o inventores desarrollen nuevos estudios. Por consiguiente, sólo unos pocos países en desarrollo tendrían los recursos necesarios para aumentar sus niveles de investigación y desarrollo. 
For a world simple we find that stronger intellectual property laws have a positive impact on a country's ability to expand its productive frontier and apply tacit and explicit innovative advances. However, this effect is restricted to countries with an above average level of development and complexity. For developing countries our results show that IPRs have a best non-significant effect on economic complexity and might even have a negative effect on these countries' ECI (Mehlig y Eterovic, 2015, p. 674).

Las disposiciones de los ADPIC ofrecen pocas garantías directas de que habrá un aumento de la transferencia tecnológica a los países pobres. Por lo tanto, la transferencia de tecnología como efecto de fuertes sistemas de derechos de propiedad intelectual es teórica porque depende de muchos factores que están estrechamente relacionados con la realidad política, social y económica del país: "TRIPS recognizes in Article 7 that the transfer and dissemination of technology is a fundamental objective of the global IPR system. However, most provisions of TRIPS offer little direct assurance that there will be a rise in ITT to poor countries" (Maskus, 2004 , p. 3). Los actores de los países en desarrollo deben intentar aprender y asimilar nuevas tecnologías y encontrar una aplicación que pueda ser útil de acuerdo con sus necesidades e intereses. Por otro lado, los titulares de propiedad intelectual deben tener la voluntad de enseñar a estas personas cómo utilizar la tecnología disponible. Sin embargo, estas son metas difíciles de alcanzar por múltiples razones. Según Maskus,

Many developing countries have complained for a long time that the flows of ITT through private channels are inadequate for their competitive and social needs. Implicitly the claim is that the volume (and quality) of technology transfers is well below optimal. In principle, this deficiency could be the result of failures in private markets for technology, failures in surrounding factor and product markets, and failures in public policy. All of these are important reasons for limited ITT, perhaps especially the latter two as they may establish an uninviting climate for FDI and licensing (2004, p. 15).

Por otro lado, los derechos de propiedad intelectual están vinculados a un aumento de los niveles de inversión extranjera directa. Es cierto que las corporaciones transnacionales buscan invertir en países que ofrecen la misma protección de propiedad intelectual que pueden obtener en sus países. Sin embargo "existing research suggests that countries that strengthen their IPR regimes are unlikely to expertise a sudden boost in flows of FDI” (Fink y Maskus, 2005, p. 8). Esto se debe a que el nivel de derechos de propiedad intelectual no es el único elemento que los inversionistas consideran cuando toman esta decisión. Al final, normalmente toman en cuenta las condiciones generales del país con respecto a temas como la estabilidad política, la seguridad jurídica y el clima global de inversión.

Por esta razón, algunos autores sugieren que "developing country never gains from stronger intellectual property regimes until it is ready to engage in research and development at the frontiers of knowledge" (La Croix, 1995, p. 4). Por lo tanto, el bienestar y el crecimiento económico no sólo dependen del fortalecimiento de los derechos de propiedad intelectual, sino de las características y condiciones de cada país. Es importante señalar que los derechos de propiedad intelectual no son una solución para transformar un país en desarrollo en uno desarrollado. Este sistema es una herramienta que debe ser utilizada de acuerdo a las potencialidades, capacidades y recursos de cada nación. De ahí que: "the significance of intellectual property rights in economic activity differs across countries and depends (1) on the amount of resources countries devote to creating intellectual assets as well as (2) the amount of protected knowledge and information used in production and consumption" (Primo Braga, Fink y Sepulveda, 2000, p. 12). 
A partir de estas consideraciones, el presente dossier, "Los aspectos económicos de la Propiedad Intelectual”, trae a discusión un tema tan relevante como son los aspectos económicos de la propiedad intelectual y su impacto en la economía y la innovación desde una perspectiva nacional y global. Los artículos que lo integran abordan diferentes facetas y dimensiones de los derechos de propiedad intelectual en los distintos ámbitos de la vida y de la economía. Este dossier cuenta con el importante aporte del profesor Keith Maskus, uno de los referentes mundiales en este tema, a cuyas reflexiones se suman las contribuciones de distinguidos juristas internacionales y nacionales que plantean sus perspectivas ante distintas aplicaciones de los derechos de propiedad intelectual en múltiples escenarios. Con este dossier buscamos llamar al debate sobre una temática de interés general que tiene que ser llevada a cabo con estricta consideración de las realidades nacionales en función de las necesidades globales, buscando un balance entre el desarrollo económico y humano de las naciones.

\section{Referencias bibliográficas}

Abbott, F. M. (1996). Public Policy and Global Technological Integration: An introduction. Chicago Kent Law Review, 72 (2), 345-346. https://doi.org/10.1016/s02673649(97)82132-2

Biber-Klemm, S., Cottier, T. et al. (2006). The current law of plant genetic resources and traditional knowledge. En S. Biber-Klemm, T. Cottier y D.S. Berglas (eds.). Rights to Plant Genetic Resources and Traditional Knowledge: Basic Issues and Perspectives (pp. 56-111). Oxfordshire y Cambridge: Cabi.org. https://doi.org/10.1079/9780851990330.0056

Fink, C. y Maskus, K.E. (2005). Intellectual Property and Development. Washinton DC: World Bank and Oxford University Press.

Gutterman, A. S. (1993). The North-South Debate Regarding the Protection of Intellectual Property Rights. Wake Forest Law Review, 89 (28), 89-139.

La Croix. S. J. (1995). The Rise of Global Intellectual Property Rights and their impact on Asia. Asia Pacific Issues, 23, 1-8. http://scholarspace.manoa.hawaii.edu/bitstream/10125/3801/1/api023.pdf

Maskus, K. E. (2004). Encouraging International Technology Transfer. Switzerland: UNCTAD-ICTSD.

Mehlig C. y Eterovic S. (2015). Do Stronger Intellectual Property Rights Increase Innovation? World Development, vol. 66, pp.665-677. https://doi.org/10.1016/j.worlddev.2014.08.025

Primo Braga, C., Fink, C. y Sepulveda, C.P. (2000). Intellectual Property Rights and Economic Development. Washington DC: The World Bank.

Stathis, Kristi L. (2015). Ocean Tomo Releases 2015 Annual Study of Intangible Asset Market Value. Insights Blog. <http://www.oceantomo.com/blog/2015/03-05-ocean-tomo-2015-intangible-asset-market-value/>.

Wipo (2009). The Economics of Intellectual Property: Suggestions for Further Research in Developing Countries and Countries with Economies in Transition. <http://www.wipo.int/ edocs/pubdocs/en/economics/1012/wipo_pub_1012.pdfs.

\section{Acuerdos internacionales}

WTO (1994). Acuerdo sobre los Aspectos de los Derechos de Propiedad Intelectual relacionados con 
el Comercio (ADPIC). Adoptado en Marrakech el 15 de abril de 1994. <https://www. wto.org/spanish/docs_s/legal_s/27-trips.pdf>.

\section{Comunicaciones}

GATT (1990). Comunication from Argentina, Brazil, Chile, China, Colombia, Cuba, Egypt, India, Nigeria, Peru, Tanzania and Uruguay: MTN.GNG/NG11/W/71. 14 de mayo de 1990, <http://www.wto.org/gatt_docs/English/SULPDF/92100147.pdf>. 